Service social

\title{
L'évolution et la pertinence de l'approche structurelle dans le contexte social actuel
}

\section{Justin Lévesque et Jean Panet-Raymond}

Volume 43, numéro 3, 1994

Intervention individualisée et empowerment

URI : https://id.erudit.org/iderudit/706666ar

DOI : https://doi.org/10.7202/706666ar

Aller au sommaire du numéro

Éditeur(s)

École de service social de l'Université Laval

ISSN

1708-1734 (numérique)

Découvrir la revue

Citer cet article

Lévesque, J. \& Panet-Raymond, J. (1994). L'évolution et la pertinence de l'approche structurelle dans le contexte social actuel. Service social, 43(3), 23-39. https://doi.org/10.7202/706666ar
Résumé de l'article

L'approche structurelle, ainsi qu'elle a été élaborée par Maurice Moreau et les professeurs de méthodologie de l'École de service social de l'Université de Montréal, a-t-elle évolué et est-elle toujours pertinente pour la pratique des années 90 ? Si le dogmatisme idéologique s'est atténué, le contexte de la pratique justifie encore les objectifs de cette approche qui se fondent sur les postulats de l'analyse critique, la polyvalence de l'intervention et

l'augmentation du pouvoir de la clientèle. Les objectifs de pratique nécessitent une formation qui vise l'acquisition de compétences pertinentes par rapport aux postulats. 


\title{
L'évolution et la pertinence de l'approche structurelle dans le contexte social actuel
}

\author{
Justin LÉVESQUE \\ Jean PANET-RAYMOND \\ Professeurs de méthodologie
} École de service social, Université de Montréal

Le développement de I'approche structurelle (Moreau, 1979, 1982, 1987) a été longtemps associé à une période caractérisée davantage par le militantisme et l'engagement politique ${ }^{1}$. Les intervenantes ${ }^{2}$ devaient se situer, montrer leurs couleurs, dénoncer les injustices sociales, s'engager, mobiliser les ressources et remettre en question les structures établies. En prônant la mobilisation collective, en dénonçant les structures opprimantes du système, en travaillant à réduire la distance sociale entre les individus et en s'élevant contre I'idéologie dominante, l'approche structurelle s'inscrivait dans la ligne de pensée des années 70 et 80 et correspondait bien au climat de l'époque.

Ces principes associés davantage à la transformation sociale et au questionnement autour des structures institutionnelles ont parfois 
mis en veilleuse les gestes professionnels que l'intervenante structurelle posait dans sa pratique de tous les jours. D'une certaine façon, I'approche structurelle a besoin d'être démystifiée et ses hypothèses de travail davantage opérationnalisées. Dans un contexte sociojuridique tel que celui de la protection de la jeunesse, comment peut-on, par exemple, parler de relation dialogique avec les familles, alors que l'intervention privilégiée est liée au contrôle social? Comment peut-on établir une alliance stratégique avec les clients lorsque les règles de fonctionnement de l'organisme ne le permettent pas toujours?

Nous tenterons de répondre à ces questions en nous référant au contexte actuel de la pratique au moment où les principaux enjeux politiques ne sont plus les mêmes et où les conditions de la pratique et de l'enseignement ont évolué. Par exemple, dans les rapports sociaux, la tendance n'est plus à la confrontation, mais plutôt à la recherche du consensus, de la concertation, du partenariat et de l'équilibre entre les différents systèmes en jeu. La question que I'on se pose est la suivante: I'approche structurelle a-t-elle perdu sa raison d'être et sa pertinence dans la conjoncture actuelle? Nous faisons I'hypothèse que la pertinence de la vision structurelle demeure et est aussi justifiée en 1994 qu'elle l'était en 1980. Nous pensons que le libellé de l'approche et les concepts radicaux qui semblaient la distinguer des pratiques traditionnelles n'ont plus le même impact sur les intervenantes sociales. L'approche structurelle tente de faire le pont entre les croyances idéologiques et l'action. Elle reconnaît l'impact des déterminants socio-économiques sur la réalité sociale des clientèles et notamment sur les familles. Les praticiens et formateurs sont à la recherche d'un principe intégrateur d'une approche globale qui tienne compte à la fois de la dynamique psychosociale et du contexte socio-économique, culturel et politique dans lequel évoluent les familles en difficulté.

L'importance pour l'approche structurelle de s'ajuster à une réalité sociale changeante ayant déjà été discutée par plusieurs auteurs (Moreau, 1987 ; Panet-Raymond, 1988), nous nous proposons d'abord de rappeler quelques principes et postulats de base pour une pratique structurelle. Nous résumerons ensuite les principaux objectifs de l'approche structurelle et tenterons de situer leur pertinence dans le contexte actuel de la pratique sociale. Puis, nous présenterons quelques éléments de la conjoncture et quelques grandes tendances qui semblent les plus déterminants pour la pratique. Le rapport du Groupe de travail sur les orientations de la formation universitaire en travail social (GROTOF, 1993), les travaux de Mullaly (1993) et les 
rapports de recherche «Empowerment I» (1989) et «Empowerment II » (1993) de Maurice Moreau, complétés par ses collaborateurs, nous serviront de textes de base pour appuyer notre argumentation. Finalement, en tant qu'éducateurs, nous examinerons les compétences professionnelles à acquérir pour intervenir dans une perspective structurelle.

\section{L'APPROCHE STRUCTURELLE: QUELQUES PRINCIPES DE BASE}

L'approche structurelle a d'abord été marquée par l'analyse marxiste et inspirée par l'idéologie socialiste ${ }^{3}$. Consciente que les inégalités sociales et l'accessibilité limitée aux ressources en raison du sexe, de la classe sociale, de l'ethnicité, de la religion sont généralement causées par les structures mêmes de la société capitaliste, I'approche structurelle prône une transformation sociale pour rendre ces structures plus humaines et mieux adaptées aux personnes. Dans cette perspective, le changement social passe par une modification des structures plutôt que par une adaptation de l'individu ou des familles à celles-ci. C'est ce qui distingue fondamentalement I'approche structurelle des approches traditionnelles. Travailler à modifier des structures qui contribuent à enrichir les uns et à appauvrir les autres pour en arriver à une société plus équitable et plus juste. L'option politique de démasquer le pouvoir, de dénoncer l'idéologie dominante, de nommer les sources d'oppression, de refuser d'accepter le statu quo a souvent donné mauvaise presse à I'approche structurelle dans certains établissements et fait oublier que I'intervenante structurelle doit tout autant travailler à aider les individus à retrouver leur estime de soi, à satisfaire leurs besoins fondamentaux, à gérer leurs conflits interpersonnels, émotionnels et psychologiques et à développer des interactions familiales susceptibles de favoriser l'épanouissement des individus. Est-ce que cette vision est dépassée en 1994? Est-ce qu'une modification des structures est encore souhaitable ou totalement utopique?

\section{TROIS POSTUlats POUR LA PRATIQUE}

La pratique structurelle ne peut se concevoir sans certaines hypothèses de base qui sous-tendent ou transcendent cette pratique. Nous en relevons trois, soit l'analyse critique, la polyvalence dans I'intervention et l'empowerment ${ }^{4}$. 


\section{L'analyse critique}

Essentiellement, nous réitérons que I'intervenante doit être, avant tout, guidée par une vision et une orientation basées sur une approche globale et critique qui tient compte à la fois de la dynamique psychosociale et du contexte familial, socio-économique, culturel et politique dans lequel évoluent les personnes. Les attitudes et les compétences de l'intervenante structurelle seront assujetties à cette vision. Nous soumettons que l'intervenante, pour être efficace, doit d'abord être capable d'acquérir un esprit critique à l'égard d'elle-même, des orientations nouvelles, des idéologies véhiculées par la société et ses dirigeants et ensuite de travailler à créer les meilleures conditions de vie pour la collectivité. Cette vision critique s'applique également à I'organisation qui emploie les intervenantes. Le changement organisationnel est donc une option que les travailleuses doivent envisager, toujours à la lumière des intérêts de la population servie. Elle empruntera aux autres modèles de pratique les techniques pertinentes et acquerra les attitudes qui augmenteront son efficacité professionnelle.

Nous croyons que notre tableau sur les habiletés et attitudes reliées aux cinq niveaux d'intervention privilégiés par l'approche structurelle, soit l'individu, la famille, la communauté, les petits groupes et l'institution, demeure encore pertinent dans la pratique contemporaine du travail social (Lévesque et Panet-Raymond, 1987 : 425). Nous mettions de l'avant à l'époque que l'intervenante structurelle devait se signaler par sa capacité d'écoute et son aptitude à démystifier son rôle lorsqu'il était pertinent de le faire. L'objectif poursuivi était de diminuer la distance sociale et de partager les expériences communes qui rapprochent les individus. Ces habiletés encouragent les clients à s'impliquer davantage dans la définition du problème. La transparence et la disponibilité permettent un engagement social qui peut se traduire par l'empathie sociale consistant essentiellement à ajouter une dimension sociale aux attitudes personnelles d'empathie que les intervenantes ont traditionnellement apprises. C'est l'empathie appliquée au vécu social de l'individu et basée sur le sens de la démocratie. Cette attitude aidera le client à développer un sens critique à l'égard des incidences des politiques sociales et de l'idéologie véhiculée dans la société sur sa propre vie. Cette prise de conscience des événements qui marquent les individus est un premier pas vers l'engagement du client à l'égard d'une meilleure gestion de son problème. 


\section{La polyvalence dans l'intervention}

Dans la conjoncture sociale actuelle, il nous apparaît que la polyvalence dans l'intervention est la meilleure réponse aux exigences de la pratique. Par polyvalence, nous entendons cette habileté d'intervenir à différents paliers d'organisation de la réalité sociale, tels que les individus, les couples, les familles, les petits groupes, les associations, les communautés ainsi que les petites et grandes collectivités, en se basant sur une analyse multifactorielle des situations-problèmes qui lui sont présentées. Pour l'intervenante structurelle, cette polyvalence se traduit également par une analyse globale qui tient compte des variables de classe, de sexe, d'origine ethnique, de religion, de statut familial, et de toute caractéristique qui pourrait entraîner une forme d'oppression. La polyvalence, c'est aussi une sensibilité à l'égard des dimensions individuelles et collectives des oppressions qui nécessitent des interventions tant au niveau de l'individu, de la famille que de la collectivité à laquelle appartient la population servie. L'intervenante structurelle a donc une connaissance, sinon une maîtrise, des différents modèles d'intervention touchant les individus, les familles, les petits groupes et les collectivités. La ligne directrice qui sous-tend cette polyvalence est la volonté explicite de redonner le pouvoir à la clientèle ou de lui permettre $d^{\prime}$ avoir accès à un plus grand pouvoir personnel. Cette préoccupation est constante et se retrouve dans toutes les étapes de l'intervention.

\section{L'empowerment}

Ce concept semble revenir à la mode depuis quelques années, notamment par le courant psychologique, mais aussi dans les domaines de la sociologie et des écrits sur l'action communautaire. Comme I'exprime Breton (1994), I'usage parfois abusif du terme empowerment a pour effet d'en diluer le concept et son impact. Néanmoins, elle offre cette définition qui se situe dans la perspective structurelle:

Thus empowerment is the combined result or outcome of getting involved in a conscientization or consciousnessraising process, acquiring skills, and attaining a goal of a just distribution of power, especially the power to access resources or services to which one is entitled (1994: 29).

Le concept d'empowerment est au centre de l'approche structurelle. Comme l'indiquent les titres de ses deux rapports de recherche, Moreau (1989 et 1993) place cette notion au cœur de ses préoccupations. L'augmentation du pouvoir du client et la démystification de celui des 
intervenantes sont des éléments essentiels à toute intervention psychosociale. Par ailleurs, ce concept n'est pas nouveau. Déjà, dans les années trente, Bertha Reynolds (Hartman, 1986) préconisait I'empowerment des populations opprimées pour les sortir des difficultés sociales et économiques de l'époque. Elle enjoignait aussi les travailleurs sociaux de militer à l'intérieur des mouvements sociaux en solidarité avec leurs "clientèles " pour les soutenir et participer au même changement social et politique.

D'autres auteurs, tels que Le Bossé et Lavallée ${ }^{5}$, ont exprimé que ce concept "permet de cerner une réalité importante: des personnes ayant des conditions de vie incapacitantes (chômage, pauvreté, marginalité, etc.) prennent leurs affaires en main et font avancer leur cause» (1993: 11). Les mêmes auteurs expriment que: "Replacée dans le contexte de la mise en question des pratiques traditionnelles d'intervention sociale et des modèles théoriques qui les fondent, I'idée d'une réappropriation active du pouvoir thérapeutique apparaît stimulante»(1993:11). Dans cette perspective, l'empowerment signifie essentiellement le fait de remettre le pouvoir aux personnes qui vivent une réalité sur laquelle elles ont perdu une emprise. La précarité des ressources économiques et les difficultés liées au chômage et aux conditions de vie difficiles sont particulièrement propices à la mobilisation des forces des individus pour les aider à se conscientiser à l'oppression qu'ils subissent ou qu'ils ont appris à subir.

Staples (1990) relie le concept d'empowerment à l'habileté de prendre ses décisions et d'agir pour soi-même individuellement ou collectivement. C'est une prise en charge ou une prise de contrôle sur sa propre destinée qu'il décrit en ces termes:

Thus, empowerment is egalitarian in nature, stressing the competence and right of people to take charge of their own destiny. For the powerless, this entails a bottom up process whereby they transform from passive or reactive subjects to positive actors in the drama of their individual lives (1990:31).

Cette prise en charge collective par les populations opprimées amène donc une participation à des mouvements sociaux qui luttent pour des conditions de vie meilleures et la défense des droits des individus. La pratique professionnelle dépasse donc le milieu institutionnel et exige un engagement qui peut parfois se traduire par un militantisme syndical et par des prises de position publiques. L'Ordre professionnel des travailleurs sociaux du Québec, en se prononçant récemment sur la loi 37 concernant la sécurité du revenu et en faisant ressortir les aspects injustes de cette loi, illustre bien l'importance 
de cette implication. Finalement, nous suggérons que le concept d'empowerment a des répercussions même dans la vie privée et entraîne un engagement personnel, voire une philosophie de vie (Moreau et Frosst, 1993; Mullaly, 1993).

\section{LES OBJECTIFS DE L'APPROCHE STRUCTURELLE}

Avant de situer la pertinence de l'approche structurelle, nous reverrons brièvement les quatre principaux objectifs poursuivis tout en faisant ressortir ce qui la distingue d'une pratique plus traditionnelle du travail social 6 .

- Aider le client à avoir pleins pouvoirs sur sa vie et favoriser I'acquisition de pouvoir dans ses rapports sociaux (Moreau, 1993 ; Breton, 1994 ; Le Bossé et Lavallée, 1993 ; Staples, 1990). Concrètement, cet objectif se traduit par une démystification du rôle d'expert et de professionnel pour redonner le pouvoir à ceux qui en ont été dépouillés. Les approches traditionnelles se sont souvent caractérisées par des décisions unilatérales de l'intervenante qui sait ce qui est bien pour le client et sa famille. Alors que la pratique a longtemps suggéré que le rôle de l'intervenante est de définir le problème et de placer une étiquette diagnostique sur le client, I'approche structurelle recommande une définition commune du problème où intervenante et clients associent leurs efforts pour déterminer la source du problème. Cela se traduit par une relation plus égalitaire et dialogique (Freire, 1974).

- Contribuer à la matérialisation des problèmes sociaux. Cet objectif consiste à faire le lien entre les conditions de vie existantes et le problème vécu. Ne pas psychologiser indûment les problèmes sociaux pour en faire porter le poids uniquement aux clients et travailler unilatéralement à l'adaptation des individus aux conditions opprimantes.

- Favoriser la collectivisation des problèmes. Les individus ne vivent pas seuls leurs problèmes. L'intervenante aide les personnes à briser l'isolement, encourage une prise de conscience commune de situations qui affectent tout un groupe d'individus et s'engage avec les clients à mettre en place des services alternatifs. Ici, le pouvoir du nombre est une force non négligeable. La conception traditionnelle du service social a longtemps promu la responsabilité individuelle et a conséquemment contribué à un certain isolement des clients. 
- Promouvoir la défense des droits des individus et des collectivités. La travailleuse sociale s'implique activement à trouver, rendre disponibles et créer des ressources pour ses clients; elle devient à la fois une courtière, une médiatrice, une défenseure des droits des individus. Son action ne se substitue pas cependant à la responsabilité individuelle. L'intervenante enlève les obstacles qui empêchent la cliente de décider pour elle-même. Elle travaille avec les clientes et non «à la place de»ou "sur» les clientes. Traditionnellement, l'action politique de la travailleuse sociale a souvent été associée à un militantisme considéré parfois comme indésirable pour une pratique professionnelle.

\section{LA PERTINENCE DE L'APPROCHE STRUCTURELLE}

Pour que la poursuite de ces objectifs soit encore réaliste en 1994, il importe de continuer de promouvoir les rôles que l'approche structurelle juge essentiels à une pratique sociale efficace. Par un examen du contexte actuel de la pratique, nous tenterons d'établir que les grands objectifs de l'intervention structurelle n'ont pas perdu de leur pertinence. Au moment où les conditions de vie et de travail deviennent au cœur des préoccupations sociales, il semble que les objectifs précédemment décrits prennent tout leur sens. Si on parle moins d'oppression, on parle de plus en plus de l'exclusion sociale d'une grande partie de la population. En effet, dans un contexte changeant où les services publics sont remis en question et où la recherche de solutions de remplacement d'un appareil de services devenu lourd, bureaucratique et inefficace est constante, l'approche structurelle peut offrir une perspective critique, souple et sensible aux mouvements sociaux. Ceux-ci se manifestent notamment par une volonté d'avoir une part plus importante de la distribution des services sociaux et une reconnaissance accompagnée de financement juste et équitable.

\section{QUELQUES ÉLÉMENTS DE LA CONJONCTURE DE LA PRATIQUE SOCIALE CONTEMPORAINE}

Sans vouloir être exhaustifs, il nous apparaît que certains facteurs influencent plus particulièrement le contexte de nos pratiques sociales. Nous remarquons, entre autres, un courant de néolibéralisme et une tendance à la mondialisation de l'économie. Plus près de nous, 
sur le plan institutionnel, la loi 120 amène au Québec une restructuration des services sociaux et le gouvernement ne semble pas trouver $d^{\prime}$ autres moyens que de couper dans les programmes sociaux pour diminuer les déficits financiers qui menacent son économie. Il exige que les citoyens se responsabilisent dans tous les domaines, alors qu'il se désengage vis-à-vis des initiatives jugées vitales il y a quelques années. Il en découle une mise à contribution des réseaux naturels et des organismes communautaires (Panet-Raymond et Bourque, 1991 ; Panet-Raymond, 1992).

\section{Le néolibéralisme}

Ce courant se propage, à quelques exceptions près, partout dans le monde et spécialement dans les pays industrialisés où l'État-providence a connu ses heures de gloire. Ce courant a amplifié l'impact des crises en diminuant la place de l'État qui avait eu pour rôle de protéger les citoyens contre les aléas de la vie. La réduction des mesures de sécurité économique et sociale et la promotion de l'individualisme ne font qu'accentuer la gravité de la pauvreté et de ses conséquences (Fortin, 1988 ; Langlois, 1990).

\section{La mondialisation de l'économie}

Il est difficile de comprendre les implications d'un changement structurel sur le plan individuel si on n'a pas une compréhension de la structure plus grande qui les englobe. L'économie locale est dépendante de ce qui se passe à un niveau international. La récession en Turquie, par exemple, cause une migration de travailleurs vers I'Allemagne et affecte ainsi sa main-d'œuvre et son économie qui influe en retour sur l'économie américaine qui en bout de ligne touche l'économie canadienne qui implique l'économie locale. On pourrait aussi souligner l'impact des crises politiques (Rwanda, URSS, Haïti) sur la demande de services sociaux. C'est l'effet « domino» et nous suggérons que la pratique du service social ne peut I'ignorer.

La globalisation des problèmes socio-économiques donne une ampleur et une visibilité plus grandes aux problèmes sociaux contemporains. Cela entraîne également une interrelation des phénomènes qui rend difficiles les actions isolées et plus aléatoires les mesures de prévisibilité. On ne peut plus intervenir en vase clos. Les idées et les idéologies circulent librement dans le monde. Le déplacement des populations crée des interactions entre les cultures, les croyances et les coutumes. Les valeurs traditionnelles sont bousculées et les façons 
de faire sont remises en question. Cela conduit à des tensions et à de la violence dans les rapports sociaux entre individus et entre collectivités qui manifestent leurs différences de classe, de race, d'ethnie, d'âge, de religion, de sexe, d'orientation sexuelle, de statut physique, de statut familial, etc.

Ces deux éléments de la conjoncture contribuent à l'alourdissement des problèmes, à l'appauvrissement et à l'augmentation des clientèles des services sociaux et des services de santé. Les populations vieillissent, les problèmes s'intensifient et les ressources pour y faire face diminuent.

\section{LA LOI 120 et LA POLITIQUE DE SANTÉ ET DE BIEN-ÊTRE DU QUÉBEC}

Cette loi a amené une restructuration des services sociaux et de santé et provoqué plusieurs changements qui touchent directement la pratique du travail social. La décentralisation des services, la conception de mécanismes décisionnels pour la planification régionale d'organisation de services (PROS) et la création de comités intersectoriels et multidisciplinaires pour définir des stratégies d'action concertée sont des transformations importantes qui exigent le renouvellement des pratiques. Par ailleurs, la création des CPEJ entraîne une spécialisation en "enfance" qui risque d'isoler les intervenantes dans une pratique monolithique. Cette approche plus spécialisée pourrait être compensée par une définition plus large de la santé qui inclurait les dimensions sociale, économique et culturelle (OMS) et la reconnaissance légale des organismes communautaires et de leur rôle de partenaires significatifs dans les services de santé et de bien-être du Québec.

En 1992, la politique de santé et de bien-être du Québec a élargi la définition des problèmes sociosanitaires pour donner à ceux-ci une dimension multifactorielle. Parallèlement, cette politique propose des stratégies d'action telles que le renforcement du potentiel des personnes, le soutien des milieux de vie et le développement des environnements sains et sécuritaires, l'amélioration des conditions de vie, l'action en concertation au profit des groupes les plus vulnérables, la concertation intersectorielle et l'application des solutions les plus efficaces et les moins coûteuses. Cette politique met en avant les programmes qui intensifient la lutte contre l'appauvrissement et l'exclusion sociale, qui améliorent la qualité des milieux de vie et qui apportent un soutien aux réseaux sociaux et d'entraide. 
Le rapport Bouchard (1991) ajoute des éléments importants de perspectives qui devraient influencer la pratique des intervenantes auprès des enfants et des familles plus particulièrement. On y prône une approche préventive et communautaire et on considère que la lutte à la pauvreté doit être une priorité pour aider les familles. Cela fait appel à une vision plus large qui implique la collaboration ou la création de ressources communautaires telles les maisons de familles pour développer les compétences parentales, I'entraide et I'autonomie dans une perspective de promotion de la famille.

Ces politiques sociales correspondent aux perspectives de I'approche structurelle. II ne faut pas être dupe, cependant, des effets pervers de ces politiques qui peuvent apporter des réductions importantes de financement au nom de la prise en charge et de la communautarisation.

\section{QUELQUES TENDANCES DÉTERMINANTES POUR LA PRATIQUE}

Ces tendances qui interpellent le service social et qui ont été nommées «enjeux structurants » par les auteurs du rapport du GROTOF sont, entre autres, I'appauvrissement de la population et un certain nombre de changements de cap de la société qui ont été qualifiés de virages familial, communautaire, sociojuridique et technocratique. D'autres auteurs (Lévesque et Mayer, 1994 ; Boucher, 1994 ; Favreau et Hurtubise, 1994) ayant récemment développé ces thèmes, nous nous en tiendrons à celui de la pauvreté.

\section{L'appauvrissement des individus et des familles}

La pauvreté et l'appauvrissement sont des facteurs déterminants dans la pratique sociale et font partie des arguments les plus convaincants pour la promotion de l'approche structurelle. La crise économique des années 90 a bouleversé des populations qui jadis vivaient dans la relative quiétude d'un emploi stable ou de prestations de l'État qui permettaient une certaine stabilité. Cet appauvrissement ne frappe pas tous les individus, mais il entraîne des répercussions économiques structurelles et des transformations permanentes. La pauvreté augmente et apparaît de moins en moins comme un accident de parcours, mais plutôt comme un état presque statutaire sur lequel les sujets n'ont plus d'emprise. Dramatiquement, elle fait place à la misère. Les sans-abri, souvent objet de mépris, marginaux et ignorés, font la une des journaux tant au Québec qu'ailleurs. La visibilité de 
la pauvreté ne cesse d'augmenter alors que le filet de la sécurité sociale ne suffit plus et que les politiques punitives qui prétendent pousser les gens au travail et les aider à se sortir de leur pauvreté ne se révèlent pas efficaces.

La pauvreté ne peut être définie seulement en termes économiques ou comme l'absence d'argent ou de ressources matérielles. Elle est aussi une absence de pouvoir qui se traduit par le manque de contrôle sur sa destinée. Réservée jadis à la classe la plus démunie, elle touche maintenant la classe moyenne. La pauvreté a un visage. Celui-ci est représenté par la perte de la dignité humaine, tant individuelle que collective, et la négation des droits humains fondamentaux. La pauvreté est au cœur des formes d'oppression qui justifient une approche structurelle, qui vise à expliquer et changer les conditions matérielles objectives en luttant contre les causes de la pauvreté tout en tentant de réduire les tensions immédiates. Cela se fait notamment par la déculpabilisation des personnes touchées et la collectivisation des définitions des problèmes et des enjeux: on pourra ensuite créer des ressources communautaires de dépannage ou, mieux, des ressources qui favorisent l'entraide et le développement économique, telles que les cuisines collectives et la création d'entreprises communautaires.

Quant aux familles, elles font face aux difficultés économiques et aux conditions de vie difficiles engendrées par des problèmes plus larges. La tendance est de mettre l'accent sur l'aspect préventif et éducatif au lieu d'agir uniquement lorsque la famille doit être divisée. À cet effet les propositions de promotion et de prévention faites par le rapport Bouchard (1991) sont en accord avec la vision et les objectifs de l'approche structurelle, misant beaucoup sur la lutte à la pauvreté pour réduire les problèmes de fonctionnement des familles.

L'État, cherchant à réduire sa responsabilité sociale et surtout sa participation économique à la lutte contre les problèmes sociaux, se tourne vers les communautés et espère une plus grande prise en charge de la distribution des services à ses membres. Une plus grande place est offerte aux organismes communautaires avec promesse d'un meilleur financement. En conséquence, la pratique professionnelle est ainsi influencée par une vision plus communautaire, et partant plus collectiviste et plus sensible au contexte social, économique, politique et culturel. L'entraide, le partenariat et la concertation sont les éléments clés de cette tendance. 


\section{LES COMPÉTENCES NÉCESSAIRES À L'INTERVENANTE SOCIALE DANS LES ANNÉES 90}

Les tendances déterminantes pour la pratique sociale, les répercussions des décisions gouvernementales, en particulier celles de la loi 120 , et les virages qui se dessinent dans le champ du social modifient considérablement la pratique moderne du service social et évoquent, jusqu'à un certain point, la nécessité d'un renouvellement de cette pratique. Tous ces éléments militent en faveur de l'importance pour les intervenantes d'acquérir des compétences basées sur une alliance avec le client plutôt que d'adopter une approche fondée uniquement sur l'expertise professionnelle, comme les modèles traditionnels I'ont si souvent prôné. Dans cette perspective, de nouvelles stratégies $d^{\prime}$ intervention doivent être élaborées pour faire face aux nouveaux défis. La négociation, la médiation, la gestion des conflits, le partenariat, les alliances interpersonnelles et le travail «avec » plutôt que le travail «sur» font partie des stratégies nouvelles d'intervention des travailleuses sociales. L'intervention exigera aussi une meilleure connaissance des politiques sociales et des mouvements sociaux qui tentent $\mathrm{d}^{\prime}$ améliorer ces politiques. Conformément aux codes de déontologie des ordres et associations de travailleuses sociales, les praticiennes devraient s'impliquer dans les mouvements sociaux luttant pour des changements au profit des populations les plus démunies. Cette implication soulève souvent le dilemme des intervenantes divisées entre le respect des politiques de leur employeur et les intérêts de leur clientèle (Hardina, 1994).

Le tableau synthèse de la page suivante présente, d'une façon succincte, les habiletés d'intervention associées à une pratique structurelle. Ce tableau constitue un survol des principaux éléments de I'approche structurelle. II précise les niveaux d'intervention, rappelle les postulats de base, définit les objectifs de l'intervention, présente les principales fonctions de l'intervenante, décrit les attitudes et nomme quelques-unes des techniques les plus souvent utilisées en approche structurelle.

Comme le suggère notre tableau synthèse, les habiletés d'intervention gravitent autour des quatre grands objectifs décrits précédemment, soit l'augmentation du pouvoir personnel des individus et de celui des collectivités, la matérialisation des problèmes sociaux, la collectivisation des problèmes et la défense des droits des individus et des communautés. À partir d'une évaluation faite conjointement avec le client, l'intervenante structurelle, selon les besoins, assumera les fonctions de courtier, de médiateur, de défenseur des droits des 
Tableau synthèse

de l'approche structurelle

\begin{tabular}{|c|c|c|c|c|c|}
\hline $\begin{array}{l}\text { niveaux } \\
\text { d'intervention }\end{array}$ & $\begin{array}{l}\text { postulats } \\
\text { de base }\end{array}$ & $\begin{array}{l}\text { objectifs } \\
\text { d'intervention }\end{array}$ & $\begin{array}{l}\text { fonctions } \\
\text { de l'interv. }\end{array}$ & attitudes & techniques \\
\hline $\begin{array}{l}\text { individus } \\
\text { familles } \\
\text { petits groupes } \\
\text { collectivités } \\
\text { organisations }\end{array}$ & empowerment & $\begin{array}{l}\text { 2. matérialisation } \\
\text { des problèmes } \\
\text { sociaux } \\
\text { 3. collectivisation } \\
\text { des problèmes }\end{array}$ & défenseur & $\begin{array}{l}\text { empathie sociale } \\
\text { relation dialogique } \\
\text { respect } \\
\text { sensibilité } \\
\text { flexibilité } \\
\text { ouverture d'esprit } \\
\text { soutien } \\
\text { cohérence } \\
\text { démocratie } \\
\text { transparence }\end{array}$ & $\begin{array}{l}\text { nommer les enjeux } \\
\text { écouter activement } \\
\text { recadrer le contexte } \\
\text { négocier } \\
\text { analyser } \\
\text { universaliser } \\
\text { individualiser } \\
\text { reformuler } \\
\text { confronter } \\
\text { recadrer la lecture } \\
\text { des événements }\end{array}$ \\
\hline
\end{tabular}

personnes ou de conseiller. Elle acquerra des habiletés personnelles qui lui permettront de redonner aux individus et aux collectivités le pouvoir de se prendre en main. Les techniques privilégiées ici ne sont pas exclusives à l'approche structurelle; toutefois, elles sont assujetties à une vision intégrative des problèmes sociaux.

\section{CONCLUSION}

Si la réalité sociale des années 90 n'est plus la même, les problèmes sociaux, particulièrement ceux associés aux conditions socio-économiques difficiles et à l'accroissement de la pauvreté, font ressortir la pertinence d'une approche intégrée qui vise à permettre aux individus de se prendre en main et de se mobiliser devant le désengagement de l'État et les mesures de plus en plus restrictives mises en place pour composer avec un climat économique difficile. II nous apparaît que la polyvalence des intervenantes et leur engagement social et politique correspondent davantage aux besoins des individus et des collectivités qui sont très souvent démunis face au pouvoir politique. Enfin, la lourdeur bureaucratique des institutions laisse le client pour compte et l'intervenante n'a pas le choix d'intervenir au nom du client, pour le client et avec le client. C'est cette approche structurelle révisée que nous privilégions. Fondamentalement, les principes sont demeurés les mêmes, mais la façon de les appliquer s'est adaptée aux besoins contemporains de la pratique. 
Du fait des nouvelles formes de partenariat qui se développent de plus en plus avec les organismes communautaires et même les entreprises, il va de soi que les intervenantes se doivent d'être des citoyennes actives dans leur milieu avec le plus de cohérence possible entre leur vie privée et leur vie professionnelle. La défense des droits et l'empowerment représentent sans doute des choix de vie et c'est à cela que l'approche structurelle les convie.

\section{Notes}

1. Pour une meilleure compréhension du texte, le lecteur voudra se référer à notre article "L'apprentissage d'une approche intégrée: I'expérience de l'approche structurelle », dans Service social, vol. 36, n 2-3:413-433, 1987. Le présent article est un regard sur I'approche structurelle sept ans plus tard dans une conjoncture sociale bien différente.

2. Pour faciliter la lecture et être fidèles à notre principe personnel d'alternance dans les articles, nous utiliserons le féminin pour désigner les travailleurs sociaux et les travailleuses sociales. Dans notre dernier article, nous avions utilisé le masculin.

3. Le lecteur voudra se reporter au chapitre 5 de Mishra Ramesh «Society and Social Policy » et au chapitre 6 "The Marxist Paradigm», dans R. Mullaly, Structural Social Work: Ideology, Theory, and Practice, pour une présentation de la perspective marxiste du bien-être social et la conception d'une société juste.

4. Nous avons décidé de ne pas traduire en français le mot empowerment. Pour nous, il veut dire «le fait de permettre au client d'actualiser son pouvoir ou de prendre du pouvoir ».

5. Le Bossé et Lavallée (1993: 11) rapportent que « cette réalité a été perçue par plusieurs auteurs tels Rappaport (1987) comme une véritable alternative à la prise en charge clinique des difficultés psychologiques rencontrées par les individus. La notion d'empowerment comporte donc une dimension d'affranchissement individuel et collectif qui ne se limite pas au champ de la santé mentale », mais peut s'appliquer à la grande majorité des secteurs d'intérêts associés aux sciences humaines. Ces auteurs décrivent la proposition de Rappaport et résument ainsi ses deux principales affirmations: "La première est que la réalité, recouverte par ce concept, constitue un objectif souhaitable pour l'ensemble de la population et plus particulièrement pour les minorités. La seconde est que le système social à privilégier est celui dans lequel les personnes exercent un contrôle direct sur les décisions et événements qui ont un impact sur leur vie quotidienne»(1993 : 12). Cette idée d'une réappropriation active du pouvoir individuel, familial et collectif nous apparaît stimulante.

6. Dans son rapport Empowerment Through a Structural Approach to Social Work, Moreau a analysé la pratique de cent travailleurs sociaux qui avaient été formés à l'approche structurelle à l'université Carleton. L'auteur avait pour objectif d'évaluer la spécificité de l'intervention et de jauger l'impact d'une orientation structurelle sur la pratique du travail social. À partir d'un questionnaire basé sur les quatre objectifs principaux de l'approche, soit l'augmentation du pouvoir du client, la matérialisation des problèmes sociaux, la collectivisation des problèmes sociaux et la défense des clients, on fait I'analyse des actes faits par I'intervenante structurelle. Dans Empowerment II, les auteurs poursuivent le travail de Moreau et présentent une analyse de la pratique structurelle auprès de 31 praticiens. L'objectif de cette étude est d'explorer la signification d'une pratique structurelle et son opérationalisation dans la pratique quotidienne du service social. Dans Structural Social Work: Maurice Moreau's Challenge to Social Work Practice, Ben Carniol (1992) examine le concept d'empowerment et évalue la contribution de l'approche structurelle au développement d'une pratique non traditionnelle en service social. 


\section{Références bibliographiques}

BOUCHER, Laurier (1994). "La réforme et les services sociaux aux jeunes", Intervention, juin, $\mathrm{n}^{\circ} 98: 7-17$

Breton, M. (1994). "Relating Competence, Promotion and Empowerment», Journal of Progressive Human Services, vol. 5, $\mathrm{n}^{\circ} 1$.

CARNIOL, Ben (1992). "Structural Social Work: Maurice Moreau's Challenge to Social Work Practice», Journal of Progressive Human Services, vol. 3, $\mathrm{n}^{\circ} 1$.

FAVREAU, Louis et Y. HURTUBISE (1994). "Les CLSC dans les communautés locales : quelle action communautaire à I'heure de la réforme Côté? », Intervention, juin, $\mathrm{n}^{\circ} 98: 42-53$.

FORTIN, Denis (1988). Riches contre pauvres. Québec: Les Éditions autogestionnaires.

Freire, P. (1974). Pédagogie des opprimés. Paris: Maspero.

GROUPE DE TRAVAIL POUR LES JEUNES (1991). Un Québec fou de ses enfants (rapport Bouchard). Ministère de la Santé et des Services sociaux, Québec.

HARTMAN, A. (1986). "The Life and Work of Bertha Reynolds : Implications for Education and Practice Today», Smith College Studies in Social Work, $56: 79-94$.

HARDINA, D. (1994). "Social Action and the Canadian Social Worker: A Study in the Political Economy of the Profession », Journal of Community Practice, vol. 1, $\mathrm{n}^{\circ} 2: 113-130$.

LANGLOIS, Richard (1990). S'appauvrir dans un pays riche. Montréal: CEQ et Éditions Saint-Martin.

LE Bossé, Y.D. et M. LAVAlLÉE (1993). «Empowerment et psychologie communautaire ; aperçu historique et perspectives d'avenir », Les Cahiers internationaux de psychologie sociale, $\mathrm{n}^{\circ} 18: 7-20$.

LÉVESQUE J. et J. PANET-RAYMOND (1987). "L'apprentissage d'une approche intégrée: I'expérience de I'approche structurelle», Service social, vol. 36, $\mathrm{n}^{\circ} 2-3: 413-433$.

LÉVESQUE J. et R. MAYER (1994). L'État-providence à bout de souffle?, Intervention, juin, $\mathrm{n}^{\circ} 98: 32-41$.

Mishra Ramesh (1981). Society and Social Policy, $2^{\mathrm{e}}$ éd. Atlantic Highlands, NJ : Humanities Press.

Moreau, M. (1979). "A Structural Approach To Social Work Practice», Canadian Journal of Social Work Education, vol. 5, $\mathrm{n}^{\circ} 1:$ 78-94.

MOREAU, M. (1982). "L'approche structurelle familiale en service social: le résultat d'un itinéraire critique », Revue internationale en action communautaire, vol. 7, $\mathrm{n}^{\circ} 47: 159-171$.

MOREAU, M. (1987). "L'approche structurelle en travail social : implications pratiques d'une approche intégrée conflictuelle», Service social, vol. 36, $\mathrm{n}^{\circ} 2-3: 227-247$.

MOREAU, M. et Lynn LeONARD (1989). Empowerment Through a Structural Approach to Social Work. A Report from Practice. Rapport de recherche, École de service social de I'Université de Montréal et Carleton University School of Social Work. 
Moreau, M., S. Frosst et al. (1993). Empowerment II : Snapshots of the Structural Approach in Action. Rapport de recherche, École de service social de I'Université de Montréal et Carleton University School of Social Work. Carleton University Press.

MulLALY, Robert (1993). Structural Social Work: Ideology, Theory and Practice. Toronto: McClelland \& Stewart Inc.

PANET-RAYMOND, Jean (1988). "L'enseignement du changement politique dans un contexte politique changeant». Communication au $24^{\mathrm{e}}$ congrès international de l'Association internationale des Écoles de service social, Vienne.

PANet-RAymond, J. et D. Bourque (1991). Partenariat ou Pater-nariat?, Rapport de recherche, École de service social, Université de Montréal.

RAPPAPORT, J. (1987). "Terms of empowerment / exemplars of prevention: toward a theory for community psychology», American Journal of Community Psychology, vol. 15, n 5 : 121-144.

StAPles, Lee H. (1990). "Powerful Ideas about Empowerment», Administration in Social Work, The Haworth Press, vol. 14, $\mathrm{n}^{\circ} 2$ : 29-42.

RUFUTS (1993). Les orientations de la formation en travail social. Groupe de travail sur les orientations de la formation universitaire en travail social (GROTOF), mai. Document non publié. 